\title{
RHIC pC CNI Polarimeter: Status and Performance from the First Collider Run:
}

\author{
O. Jinnouchi*, I.G. Alekseev ${ }^{\dagger}$, L.C. Bland ${ }^{* *}$, A. Bravar**, G. Bunce ${ }^{* *}$, \\ R. Cadman ${ }^{\S}$, A. Deshpande $\ddagger$, S. Dhawan $\rrbracket$, D.E. Fields ${ }^{\sharp}$, H. Huang**, \\ V. Hughes $₫$, G. Igo ${ }^{\dagger \dagger}$, K. Imai ${ }^{\ddagger \dagger}$, V.P. Kanavets ${ }^{\dagger}$, J. Kiryluk ${ }^{\dagger \dagger}$, K. Kurita ${ }^{\S \S \ddagger}$, \\ Z. Li ${ }^{* *}$, W. Lozowski ${ }^{\text {IT }}$, W.W. MacKay ${ }^{* *}$, Y. Makdisi**, A. Ogawa**, \\ S. Rescia**, T. Roser**, N. Saito ${ }^{* \ddagger}$, H. Spinka ${ }^{\S}$, B. Surrow** D.N. Svirida ${ }^{*}$, \\ J. Tojo*, D. Underwood ${ }^{\S}$ and J. Wood ${ }^{\dagger \dagger}$ \\ ${ }^{*}$ RIKEN (The Institute of Physical and Chemical Research), Wako, Saitama 351-0198, Japan \\ $\dagger$ Institute for Theoretical and Experimental Physics, B. Cheremushkinskaya 25, Moscow, 117259, \\ Russia \\ ${ }^{* *}$ Brookhaven National Laboratory, Upton, NY 11973, USA \\ ${ }^{\ddagger}$ RIKEN BNL Research Center, Upton, NY 11973, USA \\ ${ }^{\S}$ Argonne National Laboratory, Argonne, IL 60439, USA \\ Tale University, New Haven, CT 06511, USA \\ "University of New Mexico, Albuquerque, NM 87131, USA \\ †े UCLA, Los Angeles, CA 90095, USA \\ $¥$ Kyoto University, Kyoto 606-8502, Japan \\ $\S \S$ Rikkyo University, Toshima-ku, Tolyo 171-8501, Japan \\ १IIndiana University Cyclotron Facility, Bloomington, IN 47405, USA
}

\begin{abstract}
Polarimeters using the proton carbon elastic scattering process in Coulomb Nuclear Interference (CNI) region were installed in two RHIC rings. Polarization measurements were successfully carried out with the high energy polarized proton beams for the first polarized pp collision run. The physics principles, performance, and polarization measurements are presented.
\end{abstract}

\section{INTRODUCTION}

The RHIC polarimeters play a key role in the RHIC spin program, providing fast feed-back to the accelerator, and providing measurements of the beam polarization to the experiments. In planning for the RHIC spin program, it was recognized that the traditional method of proton polarimetry at intermediate energy (at the AGS, for example) would not be sufficiently sensitive at RHIC energies. This method uses the empirical analyzing power of proton-proton elastic scattering at $-t=0.15(\mathrm{GeV} / \mathrm{c})^{2}$, where there is an observed maximum independent of energy. This analyzing power falls as $1 / p_{\text {beam }}$, and is about $1 \%$ at the RHIC injection energy of $24 \mathrm{GeV} / \mathrm{c}$.

For RHIC polarimetry, we have selected the reaction proton-carbon elastic scattering at very small momentum transfer, $-t=0.006$ to $0.03(\mathrm{GeV} / \mathrm{c})^{2}$. This $t$-range is in the Coulomb nuclear interference region (CNI), where quantum electrodynamics predicts a significant analyzing power at the peak (0.04), which is essentially constant over the entire RHIC energy range. The figure of merit for proton-carbon CNI scattering, cross-

*Work performed under the auspices of U.S. Department of Energy. CP675, Spin 2002: 15th Int'l. Spin Physics Symposium and Workshop on Polarized Electron Sources and Polarimeters, edited by Y. I. Makdisi, A. U. Luccio, and W. W. MacKay (C) 2003 American Institute of Physics 0-7354-0136-5/03/\$20.00 
section $\times$ (analyzing power) ${ }^{2}$, becomes greater than the traditional proton-proton elastic scattering polarimeter for energies greater than about $5 \mathrm{GeV} / \mathrm{c}$.

The QED prediction for the CNI analyzing power is based on the interference of the electromagnetic spin flip amplitude, which generates the proton's anomalous magnetic moment, and the hadronic non-flip amplitude. This QED prediction is modified by a potential contribution from an hadronic spin flip amplitude which is not presently calculable. Indeed, from our initial studies of this method of polarimetry near the RHIC injection energy, we have found a significantly lower analyzing power than that predicted from QED alone. The hadronic spin-flip amplitude is expected to diminish with increasing energy, so we expect to have a reasonable sensitivity for polarization measurement over the RHIC energy range, possibly increasing with energy.

The theoretical uncertainty from the unknown hadronic spin flip amplitude leads to the requirement that the proton-carbon CNI polarimeters must be experimentally calibrated over the RHIC energy range. At $22 \mathrm{GeV} / \mathrm{c}$ this has been done to $\pm 31 \%$, by making simultaneous measurements of the CNI asymmetry, and the beam polarization using a proton-proton elastic polarimeter. This calibration is ultimately tied to protonproton elastic measurements with a polarized proton target where the target polarization was measured independently. It is planned for the near future to calibrate the CNI polarimeters at RHIC at higher energy by measuring the beam polarization at injection ( 24 $\mathrm{GeV} / \mathrm{c}$ ), accelerating to a new energy, measuring the $\mathrm{CNI}$ asymmetry, then decelerating back to $24 \mathrm{GeV} / \mathrm{c}$. If the polarization is found to be the same at $24 \mathrm{GeV} / \mathrm{c}$, before and after the down-ramp, we can use this polarization value at the new energy to calculate the analyzing power. If we measure a lower polarization after the down-ramp, we will develop bounds on the analyzing power at the new energy.

For the year 2004 we plan to install a polarized hydrogen jet target in RHIC. The target polarization will be measured to about $2 \%$ using a Breit-Rabi polarimeter. The knowledge of the target polarization will be transfered to the beam polarization using proton-proton elastic scattering in the CNI region. For p-p elastic scattering, the analyzing power is the same, whether the beam or target is polarized. The beam polarization is then used to calibrate the RHIC proton-carbon polarimeters. Our plan is to reach a $\pm 5 \%$ calibration of the p-C CNI analyzing power, by 2005.

We chose proton-carbon CNI over proton-proton CNI for the RHIC polarimetry because an ultra-thin carbon ribbon target was available [1], which would allow the low energy carbon recoils to escape the target. The target would survive heating from the RHIC beam, provide sufficient luminosity for a quick precision polarization measurement, and be sufficiently thin to avoid pile-up of events in the detector at the same time. For a thicker target, only surface scattering would produce observable recoils in the CNI region, and the target would fail on the other points as well. A hydro-carbon target, for p-p CNI, would not survive the RHIC beam. A polarized hydrogen jet target, which will be used for calibration, is technically difficult, and is very thin. The luminosity available for a polarized jet is orders of magnitude less, and not practical to use as a fast polarimeter. A polarimeter based on an unpolarized hydrogen jet was considered, but is also technically complicated, without providing a major advantage over proton-carbon CNI.

The choice of CNI scattering offers several very clean experimental advantages. The recoil (carbon for the RHIC polarimeters, proton for the polarized jet calibration 


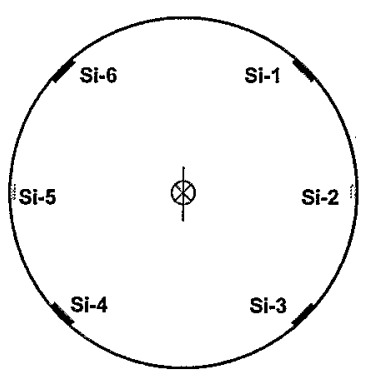

FIGURE 1. The schematic geometry layout of the silicon detectors inside the $15 \mathrm{~cm}$ radius RHIC beam vacuum pipe. The polarized proton beam direction is into the paper, and the carbon target is represented by the vertical line at the center of the vacuum pipe.

experiment) exits at nearly $90^{\circ}$ to the beam, an angle which changes only slowly with energy. Therefore, the same recoil detectors cover the entire RHIC energy range. Furthermore, the recoils are at very low energy so that a time of flight measurement can be made, relative to the rf-bunched beam. The relationship between the velocity and energy of the recoil identifies the mass of the recoil. The CNI reaction is two orders of magnitude larger in cross section than nearby inelastic reactions. Finally, the slow recoil arrives at the detectors well after prompt background, which is timed with the rf-bunched beam.

In the following sections we describe the RHIC polarimeters and the measurements during the 2001-2002 RHIC run, the first run of the first polarized proton collider.

\section{EXPERIMENTAL SETUP}

The RHIC polarimeters are located near the 12 o'clock intersection region, with separate polarimeters in each beam, referred to as the Blue and Yellow beams. A schematic of the polarimeters is shown in Fig. 1. The RHIC polarized proton beam passes through an ultra-thin carbon ribbon target, and carbon recoils from CNI scattering are observed in six silicon strip detectors placed as shown.

Very thin carbon ribbon targets have been developed at IUCF [1]. A typical target is $2.5 \mathrm{~cm}$ long, $3.5-\mu \mathrm{g} / \mathrm{cm}^{2}$ thick $(150 \AA)$ and $5-\mu m$ wide. The target is mounted on a mechanism which rotates into the beam, with a choice of 3 vertical and 3 horizontal targets. The silicon detectors have twelve $12 \mathrm{~mm} \times 2 \mathrm{~mm}$ strips, for a $24 \mathrm{~mm}$ total width. The six detectors are mounted inside of the vacuum chamber with readout preamplifier boards directly attached to the chamber detector ports through vacuum feedthrough connectors. Figure 2 shows a scatter plot of time of flight versus energy for one silicon strip in the polarimeter. The silicon detectors are $15 \mathrm{~cm}$ from the target, and the RHIC bunch length was about $3 n s$ for this run, which is from the commissioning run in 2000 [2]. The inset in the figure shows the mass distribution derived from velocity and energy (note that the Time-of-flight in the figure includes an offset of $40 \mathrm{~ns}$ ). The carbon and $\alpha$ peaks are clear, with little background under the carbon peak. 


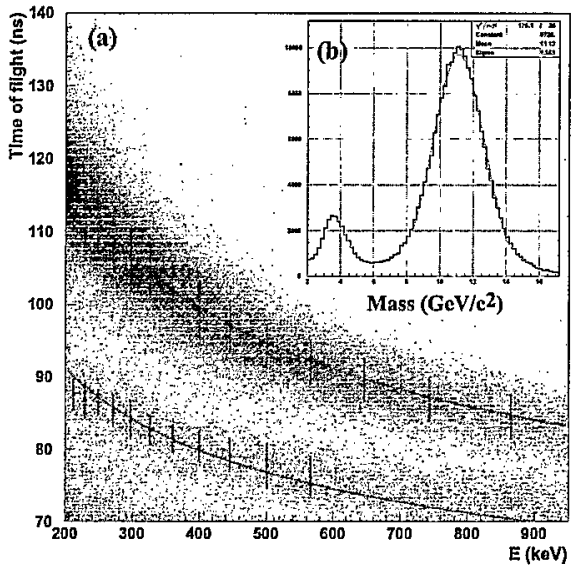

FIGURE 2. (a) The time of flight is plotted as a function of kinematic energy of the detected particle. (b) Sub-figure shows the projected mass distribution. A Carbon mass peak $\left(11.18 \mathrm{GeV} / \mathrm{c}^{2}\right)$ is clearly separated from an alpha mass peak $\left(3.7 \mathrm{GeV} / \mathrm{c}^{2}\right)$.

The beam polarization is measured by counting the number of events in the carbon band in each strip versus the azimuthal angle of the strip around the beam (Fig. 1). A vertical polarization generates a left-right asymmetry in the detectors and a radial polarization generates an up-down asymmetry.

The rates are very high, so we chose a readout system without dead time based on wave form digitizers (WFDs) [3]. The WFDs consist of a high frequency video ADC chip (used for laptop screens) and a Xilinx FPGA. The waveform from each strip was digitized every $2.4 n s$, and pulse height and time of flight, compared to the RHIC if clock, was determined in real time, and compared to a look up table which accepted the carbon band (as in Fig. 2). On-board scalers kept the number of events for each strip, and for each beam bunch. The 55 beam bunches of polarized protons in RHIC for the 2001/2 run, spaced $212 n s$ apart, alternated in polarization sign. Therefore, the on-board scalers collected data for both signs, and for bunches set up with zero polarization, for each strip. The WFDs were introduced for the 2001/2 run and 48 strips were read out, 8 for each detector (Fig. 1). Also, the orientation of the strips for the left and right detectors (Fig. 1) were set up with the strips perpendicular to the beam direction, to measure scattering angle. The $45^{\circ}$ detectors were oriented along the beam direction to reduce the azimuthal acceptance for each strip, reducing the rate compared to the $90^{\circ}$ central strips. For the $2001 / 2$ run, we typically had $4 \times 10^{12}$ protons in each ring, and $2 \times 10^{7}$ carbon elastic events were collected in about 20 seconds, with the target then rotated out of the beam. The data were then transfered to a PC, the asymmetry and various monitor asymmetries were calculated, and the result was sent automatically to the accelerator and experiments in minutes. A detailed description is given in [3], including results from a dedicated polarimeter run. 

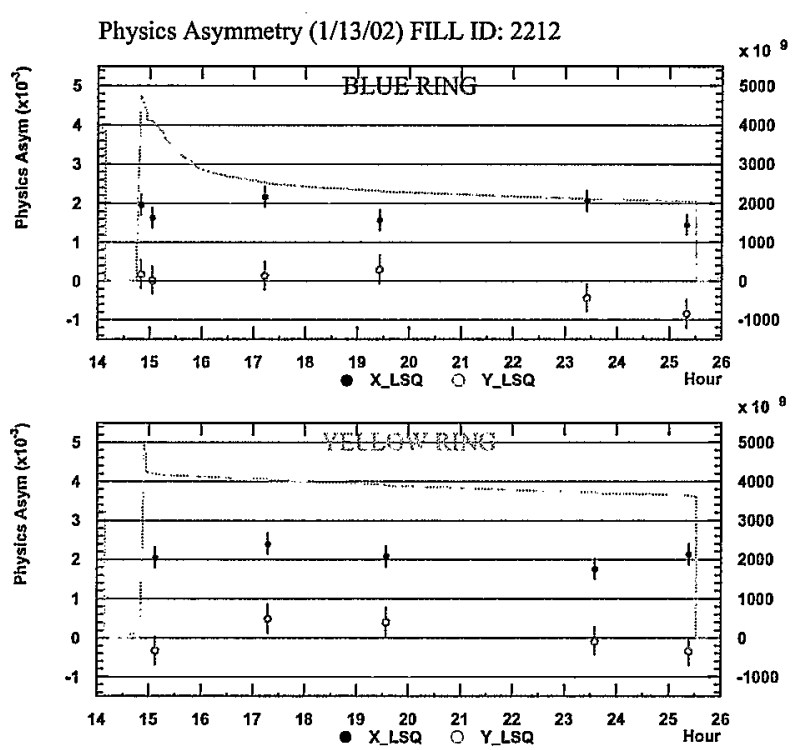

FIGURE 3. Measured physics asymmetries along with the polarized proton intensity as a function of the beam lifetime for a typical fill (Jan. 13th, 2002 fill 2212). Upper (Lower) figure shows Blue (Yellow) ring. Closed points represent the vertical asymmetry $\varepsilon_{X}$ and the open points show the radial components $\varepsilon_{Y}$; the scale is found at left axis. All the measurements are taken at $100 \mathrm{GeV} / \mathrm{c}$ store except for the first Blue measurement, i.e. run 669 was taken at injection energy. The solid curves represent the number of total protons in the ring with the right-handed axis displaying its intensity scale.

\section{POLARIMETER PERFORMANCE}

Figure 3 shows polarization measurements for a typical fill. We plot the least square fit to the data for the six detectors (48 strips), with both + and - polarized bunches. The plot shows the raw physics asymmetry, referred to later as $\varepsilon$, uncorrected for analyzing power. Either a vertical polarization is assumed, with a $(\sin \phi)$ dependence, or a radial polarization with a $(\cos \phi)$ dependence. Acceptance and luminosity asymmetries generally cancel in this fit. Ordinarily the measurements were taken at injection energy, right after the acceleration to $100 \mathrm{GeV} / \mathrm{c}$, then every two hours during the store at flattop. In some cases the injection energy measurements for the Yellow ring were omitted in order to expedite the acceleration. Although the intensity dropped during the store, the vertical polarization asymmetries (closed points) were non-zero and stable. The radial polarization asymmetries (open points), which should be zero, fluctuated around zero. Each measurement point contains $2 \times 10^{7}$ carbon events corresponding to approximately $10 \%$ relative error for a $20 \%$ polarization.

Many systematic error studies have been carried out. Referring to Fig. 1 , the $90^{\circ}$ detectors are sensitive to vertical polarization, and the $45^{\circ}$ detectors can be used to measure vertical polarization (left-right asymmetry) and radial polarization (up-down asymmetry).

The results for left-right asymmetry between the $90^{\circ}$ and $45^{\circ}$ detectors were compared 


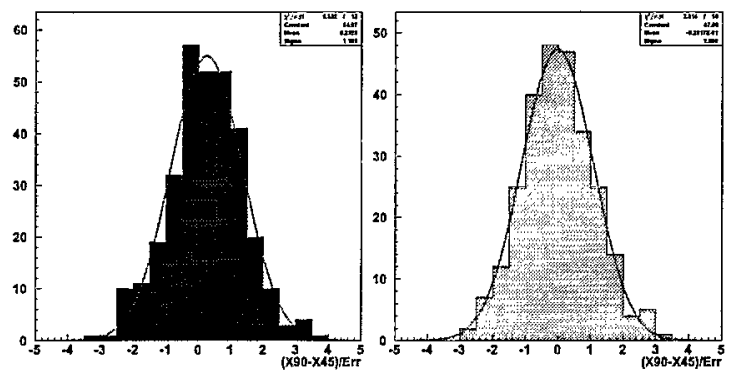

FIGURE 4. Distributions of the difference of asymmetries measured by the $90^{\circ}$ and the $45^{\circ}$ detectors. Left (Right) figure shows a Blue (Yellow) ring.

(after correction by $\sqrt{2}$ for the smaller analyzing power of the $45^{\circ}$ detectors). These were calculated using a square root formula which cancels luminosity asymmetries for + and - polarized bunches, and cancels left-right or up-down detector asymmetries, to third order [4].

The distributions for the measurements for the two beams of this difference of asymmetries, divided by the overall statistical error of the difference, is shown in Fig. 4. A Gaussian fit of the distribution gives $\overline{\varepsilon_{90-45} / \sigma_{\text {stat. }}}=0.25, \sigma_{\text {dist. }}=1.11$ for the Blue beam measurements, and $\overline{\varepsilon_{90-45} / \sigma_{\text {stat. }}}=-0.02, \sigma_{\text {dist. }}=1.10$ for the Yellow beam. We also measured up-down asymmetries, using the $45^{\circ}$ detectors. Since no radial polarization was expected (with two Siberian Snakes per ring, the stable polarization direction is vertical), the up-down asymmetry directly measures a false asymmetry. A Gaussian fit to the up-down asymmetries gives $\overline{\varepsilon_{45 \text { vert }} / \sigma_{\text {stat. }}}=0.16, \sigma_{\text {dist. }}=1.11$ for Blue and $\overline{\varepsilon_{45 v e r t} / \sigma_{\text {stat. }}}=-0.30, \sigma_{\text {dist. }}=1.43$ for Yellow. A cross asymmetry was also formed from the $45^{\circ}$ detectors, which must be a false asymmetry. This distribution gave $\overline{\varepsilon_{\text {cross }} / \sigma_{\text {stat. }}}=-0.17, \sigma_{\text {dist. }}=1.30$ for Blue and $\overline{\varepsilon_{\text {cross }} / \sigma_{\text {stat. }}}=-0.32, \sigma_{\text {dist. }}=1.26$ for $_{a}$ Yellow.

In another approach to study systematic errors, the results for different bunches were compared by normalizing the number of events in each detector for each bunch by the number of events for each detector observed for a standard or "good bunch". This normalized distribution for each bunch was fit with a (constant $+\varepsilon \times \sin \phi)$ distribution. where $\phi$ is the azimuthal position of each detector, and $\phi=0$ is vertical (Thereby allowing an asymmetry from vertical polarization). The $\chi^{2}$ for this fit showed that a small number of bunches had anomalous behavior and these bunches were removed from the polarimeter analysis. The remaining $\chi^{2}$ distribution was broader than the standard $\chi^{2}$, indicating an additional average systematic error of about $0.5 \times \sigma_{\text {statistical }}$. Anomalies for bunches were also studied by calculating specific luminosities for each bunch at the polarimeter $\left(N_{\text {total }} / I_{\text {bunch }}\right.$ where $I_{\text {bunch }}$ is the bunch current from the wall current monitor), and also at the experiments $\left(N_{\text {total }} / I_{\text {Blue }} I_{\text {Yellow }}\right)$. $N_{\text {total }}$ refers to polarimeter total counts, and to experiment counts in a luminosity monitor respectively. The anomalous bunches from these analyses matched well (for STAR, see [5]) and they were removed from the polarization and the asymmetry analyses.

Finally, the systematic error from bunch dependent effects was explored by mixing 
$(+)$ and $(-)$ bunches randomly into two groups, "+ "and " - ", each with zero (or small) polarization. These groups were used in place of the real $(+)$ and $(-)$ groups to calculate asymmetry using the square root formula. This was done 1000 times for each run, and these false asymmetries from mixed bunches are compared to the real asymmetries in Fig. 5. The false asymmetry distributions are characterized by a Gaussian with $\overline{\sigma_{\text {false }}}=1.12$ for the blue beam and $\overline{\sigma_{\text {false }}}=1.11$ for the yellow beam. The statistical error for these asymmetry measurements (both false and real) is $\sigma_{\text {stat. }}(\varepsilon)=2.8 \times 10^{-4}$.

When we compare the different studies of systematic errors in the polarimeter measurement, which are not independent, we find that each indicates a systematic error of $(0.5$ to 1$) \times \sigma_{\text {stat. }}$, for each measurement. The false asymmetries appear to fluctuate around zero, with average offsets as large as $1 / 3$ of the statistical error. We have not identified the origin of these systematic errors, and this is a work in progress.

We have decided to use, as the systematic error for the asymmetry measurement, $\sigma_{\text {systematic }}(\varepsilon)=2.8 \times 10^{-4}$. This is equal to the statistical error for a single measurement. This estimate is larger than that given in the studies above, but it reflects our uncertainty on the origin of the observed systematic errors. This systematic error is roughly $\pm 10 \%$ of the measured asymmetry. As we will discuss, the uncertainty of the analyzing power is larger than this.

We have also studied backgrounds, pile-up, and variations in the $-t$ definition from energy loss in the dead layer of the silicon. Backgrounds from inelastic reactions appear to be small $(<\mathrm{a}$ few \%), from observing the apparent signal-to-background in the carbon mass peak of Figure 2(b). Target empty data were not collected for this run, but were a small fraction in the first AGS pC CNI experiment [6], 3\%. Another background under the carbon peak, which could change with the fill or time, is de-bunched beam. In this case, the time-of-flight vs. the beam $\mathrm{rf}$ is spoiled. We studied five fills with unusually large de-bunching $(\sim 20 \%)$ and observed the effect, but also that there was little background under the carbon peak, $<$ few \%. Pile-up, two pulses in the same strip from the same crossing, can also confuse the time-of-flight vs. energy correlation. We estimated pile-up at $<1 \%$. Finally, a stable $-t$ acceptance is necessary because the analyzing power is a sharply varying function of $-t$. We estimated, based on fits for the dead-layer thickness for each silicon strip, a variation in $A_{N}$ of $< \pm 4 \%$.

We can estimate the stability of the polarimeters against varying backgrounds, pileup, or $-t$ acceptance by comparing results for the same beam for different polarimeters. This is done when we compare the $45^{\circ}$ and $90^{\circ}$ asymmetries in Fig. 4. Pile-up is also tested because the rates for the central strips for the $90^{\circ}$ detectors are about twice that of each strip for the $45^{\circ}$ detectors. Our estimated systematic error therefore includes these effects.

The analyzing power at injection energy is determined from Ref. [6], for the $-t$ ranges of the RHIC polarimeters. These were $-t=(0.7$ to 3.0$) \times 10^{-2}(\mathrm{GeV} / \mathrm{c})^{2}$ for the blue beam polarimeter, and $-t=(0.6$ to 2.7$) \times 10^{-2}(\mathrm{GeV} / \mathrm{c})^{2}$ for the yellow beam polarimeter. The difference are due to the silicon dead layer thicknesses. The values for $A_{N}$ are, $A_{N}($ blue $)=(1.27 \pm 0.40) \times 10^{-2}$ and $A_{N}($ yellow $)=(1.33 \pm 0.41) \times 10^{-2}$, where the errors are the linear additions of the statistical, raw asymmetry systematic, and beam polarization errors from Ref. [6].

Referring to Fig. 5, the measured beam polarization at injection $(24 \mathrm{GeV} / \mathrm{c})$ was, 

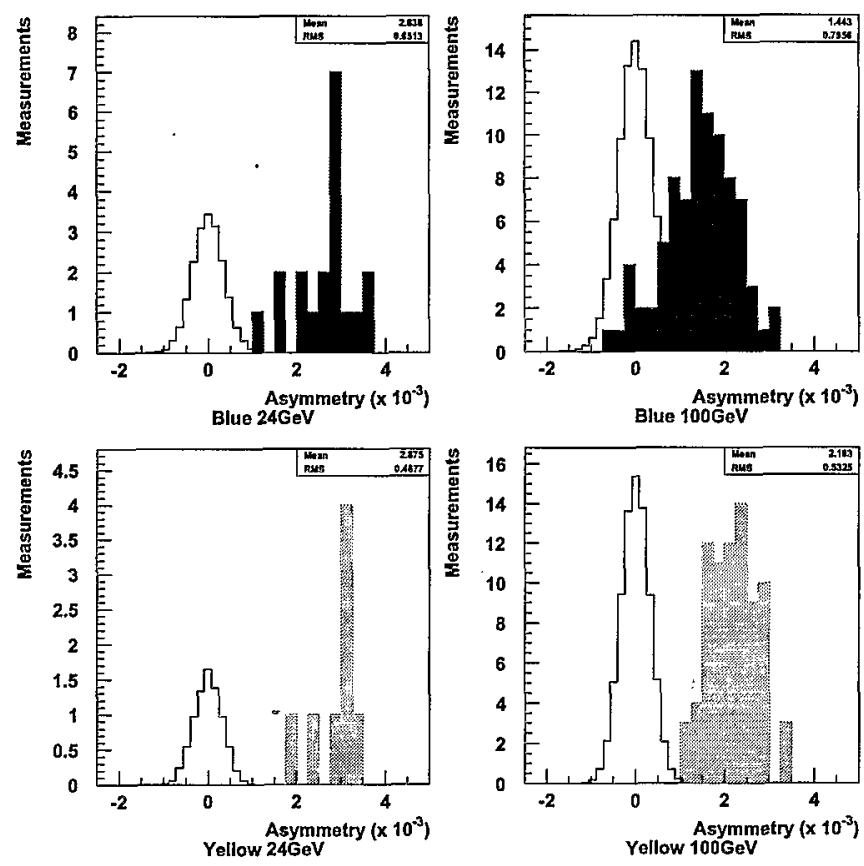

FIGURE 5. Physics asymmetries (solid histograms) are compared to the artificial asymmetries from mixed bunches (open histograms). The measurements are selected from long store ( $>4$ hours) fills after Jan. 5th. The above (below) left plot shows the asymmetries at $24 \mathrm{GeV} / \mathrm{c}$, while the right plot shows for $100 \mathrm{GeV} / \mathrm{c}$ in blue (yellow) beam.

$\overline{P_{\text {blue }}(24 \mathrm{GeV} / \mathrm{c})}=\bar{\varepsilon} / A_{N}($ blue $)=0.21 \pm 0.005 \pm 0.02 \pm 0.07$ and $\overline{P_{\text {yellow }}(24 \mathrm{GeV} / \mathrm{c})}=$ $\bar{\varepsilon} / A_{N}($ yellow $)=0.22 \pm 0.007 \pm 0.02 \pm 0.07$. The first and the second errors are the statistical and systematic errors on the raw asymmetry respectively, and the third error is a scale error from the fractional error on $A_{N}$. These results use the most stringent bunch selection, based on the polarimeters, STAR, and PHENIX data analyses of beam monitors.

We were not able to calibrate the polarimeters at $100 \mathrm{GeV} / \mathrm{c}$ for this run. There are theoretical arguments that $A_{N}$ should change only slowly with energy over the RHIC range [7]. However, an experimental calibration is required, and is planned for future runs. If we assume the same analyzing power at $100 \mathrm{GeV} / \mathrm{c}$ as for $22 \mathrm{GeV} / \mathrm{c}$, we find from Fig. $5, \overline{P_{\text {blue }}(100 \mathrm{GeV} / \mathrm{c})}=0.11 \pm 0.002 \pm 0.02 \pm 0.03$ and $\overline{P_{\text {yellow }}(100 \mathrm{GeV} / \mathrm{c})}=$ $0.16 \pm 0.002 \pm 0.02 \pm 0.05$.

\section{ISSUES AND PLANS}

There are several concerns that must be resolved before next year. One is the systematic errors that some bunches contribute to create the false asymmetry. From data, some 
bunches were observed to have unphysical ratio between six detectors. The understanding of their mechanism and criteria to discard them is needed. Another issue is the bunch by bunch polarization. Our data for the bunch by bunch analysis is limited by statistics from the previous runs. Further study on this issue is still in progress. High statistics measurements are expected for the coming run. The last concern is the serious gain drops of the silicon strip detectors. There were significantly large leakage current due to silicon radiation damage which effectively reduces the bias voltage on silicon that affects the signal shape. The replacement of the silicons and hardware improvements are planned.

For the future run, determination of the absolute analyzing power at $100 \mathrm{GeV} / \mathrm{c}$ is a crucial thing to be done, since our knowledge on the analyzing power of CNI polarimeter is limited to near the injection energy $(22 \mathrm{GeV} / \mathrm{c})$. Absolute calibration using the polarized hydrogen jet target is planned and is in preparation towards the first operation in 2004. For the moment, a calibration using a down ramp is adopted as the second best way. As a procedure, the usual polarization measurements are performed at injection, after ramp to $100 \mathrm{GeV} / \mathrm{c}$, then after down ramp again. If the polarization is found to be the same at $24 \mathrm{GeV} / \mathrm{c}$, after the down ramp vs. at injection, we can use this polarization value at $100 \mathrm{GeV} / \mathrm{c}$ to calculate the analyzing power at $100 \mathrm{GeV} / \mathrm{c}$. If we measure lower polarization after the down ramp, we will develop bounds on the analyzing power at $100 \mathrm{GeV} / \mathrm{c}$.

Finally, in the next run we hope to improve the $\mathrm{pC} C N I$ calibration by using a new $\mathrm{pC}$ CNI polarimeter in the AGS, calibrated to $p$-p elastics in the AGS internal polarimeter.

\section{SUMMARY}

The RHIC pC CNI polarimeter proved itself in the successful first polarized proton collision run 2001/2. It worked beautifully throughout the run period. Reliable high statistics $\left(20 \times 10^{6}\right.$ events) measurements were carried out in 1 minute measuring periods, owing to the successful operation of newly adopted wave form digitizer modules. The polarimeter results were broadcast to the experiments immediately after the measurements. Two Siberian snakes per ring worked well, and stable proton polarizations at $100 \mathrm{GeV} / \mathrm{c}$ were measured with little or no loss in magnitude over the store. Further detailed off-line analysis is in progress and interesting challenges are expected for the up coming run.

\section{REFERENCES}

1. W.R. Lozowski and J.D. Hudson, NIM in Physics Research, A334, 173 (1993).

2. H. Huang et al., Proc. of 2001 IEEE PAC, Chicago, p. 2443 (2001).

3. I.G. Alekseev et al. (2002), detail description is found in this proceedings.

4. G.G. Ohlsen and P.W. Keaton, NIM in Physics Research, 109, 41 (1973).

5. J. Kiryluk et al. (2002), this proceedings.

6. J. Tojo et al., Phys. Rev. Lett., 89, 052302 (2002).

7. L. Trueman (2002), RHIC Spin Note 1 hep-ph/0203013; B.Z. Kopeliovich, these proceedings. 